\title{
Análisis comparativo de los gases residuales de la combustión y fallas en motores Hyundai modelo 9h21/32, en estación de generación eléctrica distribuida
}

\section{Comparative analysis of combustion residual gases and faults in Hyundai motors model 9h21 / 32, in distributed electric generation station}

\author{
Edilberto Llanes Cedeño \\ Juan Rocha-Hoyos \\ Luis Tipanluisa \\ Giovanni Velastegui \\ Universidad Internacional SEK, Ecuador \\ Yans Guardia Puebla \\ Universidad De Granma, Cuba \\ Autor Para Correspondencia: yguardiap@udg.co.cu, antonio.llanes@uisek.edu.ec, \\ carlos.rocha@uisek.edu.ec, luis.tipanluisa@uisek.edu.ec,geovani.velastegui@uisek.edu.ec \\ Fecha de recepción: 15 de diciembre de 2016 - Fecha de aceptación: 20 de febrero de 2017
}

Resumen: El presente trabajo se realizó en la empresa de mantenimiento a los grupos electrógenos fuel - oil (emgef) en bayamo, granma - cuba. La composición de los gases fue seleccionada como indica-dores de fallas en los motores hyundai modelo 9h21/32, que componen el emplazamiento. Para la realización del experimento se utilizó un diseño multifactorial usando como factores principales 16 motores y un tiempo de trabajo de 3 meses. El cálculo y análisis periódico de los gases residuales de la combustión de los motores hyundai, se muestra en herramienta efectiva que permite obtener una valoración de la calidad de la operación en la estación. No se observaron diferencias entre los motores analizando en las composiciones de dióxido de carbono, monóxido de carbono, dióxido de azufre, relación co/co2 y eficiencia de la combustión; sin embargo, se observaron diferencias significativas entre los gases monóxido de nitrógeno y la temperatura de los gases. Las principales fallas se relacionan con fugas de gases de escape por múltiple, regulación del sistema de inyección y fugas de gases por el turbocompresor.

Palabras claves: Fallas; grupos electrógenos; gases residuales de la combustión

\begin{abstract}
: the present work was carried on in the maintenance company to generator group fuel-oil (emgef) in bayamo city, granma - cuba. The composition of the gases was selected as indicators of failures in the motors hyundai model $9 \mathrm{~h} 21 / 32$ that constitute the emplacement. For the realization of the experiment was used a multifactorial design using like essential factors 16 motors and an operating time of 3 months. Calculation and period analysis of the waste gases of the combustion of the motors hyundai, confirmed to be an effective tool that allows obtaining from the operation in the station a quality assessment. Did not observe differences between the motors analyzing the compositions of carbon dioxide, carbon monoxide, sulfur dioxide, relation co/co2 and combustion efficiency. However, significant differences between the gases nitrogen monoxide and temperature of the gases was observed. The main failures relate to leaks exhaust gas manifold, fuel injection system regulation and gas leaks by the turbocharger.
\end{abstract}

Key words: Failures; generator group; waste gases of the combustion 


\section{Introducción}

La generación de energía eléctrica consiste en transformar alguna clase de energía química, mecánica, térmica o luminosa, entre otras, en energía eléctrica. La generación eléctrica se realiza, básicamente, mediante un generador. Un generador eléctrico es un dispositivo capaz de mantener una diferencia de potencial eléctrico entre dos de sus puntos (llamados polos, terminales o bornes), transformando la energía mecánica en eléctrica. Esta transformación se consigue por la acción de un campo magnético sobre los conductores eléctricos dispuestos sobre una armadura (denominada también estator) (san miguel, 2014).

En el mundo moderno, el nivel de electrificación es un parámetro que influye en el desarrollo de un país, ya que la electricidad es la principal fuente de energía para realizar las actividades económicas. Una interrupción en el suministro eléctrico, por breve que sea, provoca considerables trastornos y pérdidas en la producción industrial, en el transporte, las comunicaciones, el sector financie-ro, entre otros. Actualmente, existe una generación mundial de energía eléctrica del orden de 20261 twh. Los principales países productores de energía eléctrica son: estados unidos, china, japón y rusia, en orden descendente; entre estos cuatro países se genera prácticamente el 50 \% de toda la energía eléctrica del planeta (fernández, 2011).

El enfoque tradicional en la generación de energía eléctrica es tener plantas centralizadas que distribuyen electricidad a través de una extensa red de transmisión y distribución. La generación distribuida (gd) proporciona la energía eléctrica en un sitio más cerca del cliente, lo que elimina los costes de transporte y distribución innecesarios. Además, puede reducir las emisiones de combustibles fósiles, aplazar el costo de capital, reducir las inversiones de mantenimiento y mejora de las condiciones en la transmisión (labis et al., 2011).

En el año 2004, cuba pone en marcha un nuevo programa que consiste en un esquema de generación eléctrica distribuida que emplea la instalación de emplazamientos compuestos por grupos electrógenos, que operan con diésel o fuel- oil. Los primeros emplazamientos instalados en el país funcionaban con combustible diésel. Sin embargo, a principio del año 2007 se anunció la conclusión oficial del programa de los grupos diésel, los cuales aportaban en conjunto más de 1300 mwh. Actualmente el programa que se está llevando a cabo es el de los grupos fuel - oil, con motores Hyundai de procedencia coreana, y man de procedencia alemana (sen, 2006).

La utilización de emplazamientos de gd con grupos electrógenos de fuel - oil lleva muy poco tiempo de explotación, y por ser una tecnología de adopción reciente en el país, no se cuenta con la experiencia y conocimientos necesarios sobre el tema, lo que conlleva al surgimiento de problemas de funcionamiento y operación de la maquinaria. Según criterios de especialistas las causas fundamentales de las averías o fallas son producto de problemas de operación, de mantenimiento, de tecnología y cultura tecnológica, lo que afecta la disponibilidad y la confiabilidad de estas plantas de generación, por lo anteriormente fundamentado el trabajo desarrollado persigue el siguiente objetivo: analizar la influencia de los gases residuales producto de la combustión, mediante la determinación de 5 tipos de gases: oxígeno, dióxido de carbono, monóxido de carbono, dióxido de azufre y monóxido de nitrógeno, como indicadores de fallas en estación de generación eléctrica distribuida. 


\section{Materiales y Métodos}

Más del $50 \%$ de la capacidad de generación eléctrica en cuba está basada en plantas gd de pequeña escala. Este es uno de los más altos índices a nivel mundial. Estas plantas generan en base a diésel y fuel - oil.

Estas tecnologías han tenido un impacto positivo en el ambiente, tienen menores tasas de consumo específico (210 g kw-1 h-1), frente a las plantas termoeléctricas basadas en la quema de petróleo crudo (284 g kw-1 h-1 en promedio). Sin embargo, la contaminación local (producción de ruido y emisiones de gases como los óxidos de nitrógenos (nox), dióxidos de azufre (so2), y de partículas de muy pequeño tamaño es un problema cuya solución está siendo estudiada. En la actualidad el mantenimiento tradicional (correctivo, preventivo) se ha visto desplazado por el mantenimiento predictivo, motivado por el surgimiento de medios de diagnóstico que permiten predecir el fallo de los sistemas.

Se han realizado estudios intensivos sobre métodos de diagnóstico para motores de combustión interna. Con el fin de detectar y diagnosticar diversos modos de fallo, tales como problemas de inyección de combustible (gertler et al., 1995; yang et al., 2001), fallos del actuador del acelerador, una deficiente combustión por fallos en el encendido (barellli et al., 2009; gardiner et al., 2007), han sido estudiados. En trabajo desarrollado por tamura et al. (2011), se ha presentado un método de detección de fallos de encendido mediante la medición de la temperatura de los gases de escape. El método propuesto es práctico para su uso en la toma de decisiones con respecto a la planificación del mantenimiento preventivo. Según weifeng et al. (2015), el contenido de nox está influenciado no sólo por la temperatura y la concentración de o2, sino también por el flujo en el cilindro y la propagación del frente de llama.

\section{Extensión de la investigación}

La investigación se desarrolló en un período de 3 meses (comprendido entre abril, mayo y junio de 2016). Dado que el universo a investigar es relativamente estrecho (16 motores), se realizó el estudio a la totalidad de la población.

La recopilación de datos e información de valor fue consultada en los siguientes documentos:

- Carta de régimen tecnológica: el hhi 1,7 mw pps. Código: uj-ig-0304

- Control de disponibilidad gdecu. Código: uj-ig 0105

- Libro de control de defecto. Código: uj-mp 0200.a5

- Libro de incidencias de operación. Código: uj-mg 0200.a8

- Control operativo de fallas.

Se realizaron consultas directas a operadores y especialistas encargados de la explotación de los grupos electrógenos (ge), extrayendo las particularidades de la funcionabilidad de los equipos. Con el fin de definir la gravedad de la falla, se ha tenido en cuenta los indicadores de gravedad referidos por daquinta (2008). Estos recogen principalmente la experiencia 
especializada obtenida en el análisis, tratamiento y consecuencias de las fallas que típicamente afectan los esquemas de control y protección.

\section{Diseño experimental}

Los resultados de los análisis y los gráficos fueron obtenidos a partir de los programas informáticos microsoft office professional plus 2013 y statgraphics centurion xV (trial versión 15.2.06, statpoint inc., usa). Para el diseño experimental fue utilizado un diseño multinivel factorial.

En el análisis de los niveles de los factores se realizó un análisis de varianza, donde se calculó un valor f para un valor de probabilidad determinado del $95 \%$ y se comparó con un valor tabulado, para así poder determinar diferencias significativas entre los niveles de los factores. Los niveles de los factores fueron definidos como sigue: el factor motor fue fijado a 16 motores, y el factor meses se evaluó en 3 meses: abril, mayo y junio.

Se analizaron 8 respuestas: oxígeno, monóxido de carbono, dióxido de azufre, monóxido de nitrógeno, temperatura de los gases, dióxido de carbono, relación co/co2 y eficiencia de la combustión. La determinación de los componentes en los gases de escape se empleó el analizador de gases telegan gas monitoring. "tempest 100”.

\section{Resultados y discusión}

\section{Varianza de los gases residuales de la combustión}

En la tabla 1, se muestra el resultado del análisis de varianza del diseño utilizado. Se observaron diferencias significativas entre los motores para el gas monóxido de nitrógeno, entre la temperatura de los gases, y para el caso del monóxido de carbono su variación resultó entre los meses, debido a que los valores de probabilidad fueron inferiores a 0,05 el cual caracteriza el $95 \%$ de probabilidad. Mientras que, no se observaron diferencias significativas entre los demás gases.

\section{Tabla 1}

Análisis de varianza de gases residuales

\begin{tabular}{lccccc}
\hline Fuente de variación & $\mathbf{s c}^{\mathbf{a}}$ & $\mathbf{g l}^{\mathbf{b}}$ & $\mathbf{C m}^{\mathbf{c}}$ & $\begin{array}{c}\text { F- } \\
\text { ratio }^{\mathbf{d}}\end{array}$ & $\begin{array}{c}\text { P- } \\
\text { valor }\end{array}$ \\
& \multicolumn{2}{c}{ Oxígeno $\left(\mathrm{o}_{2}\right)$} & & & \\
\hline a:motores & 106,99 & 15 & 7,13 & 1,06 & 0,4285 \\
\hline b:meses & 12,924 & 2 & 6,46 & 0,96 & 0,3940 \\
\hline Residual & 194,82 & 29 & 6,72 & & \\
\hline Total (corrected) & 316,46 & 46 & & & \\
\hline \multicolumn{7}{l}{ Monóxido de carbono $(\mathrm{co})$} & & \\
\hline a:motores & 40466,1 & 15 & 2697,74 & 0,72 & 0,7467 \\
\hline b:meses & 28879,8 & 2 & 14439,9 & 3,85 & $0,0330^{\mathrm{e}}$ \\
\hline Residuos & 108866,0 & 29 & 3754,0 & & \\
\hline Total (corregido) & 176511,0 & 46 & & & \\
\hline
\end{tabular}




\begin{tabular}{|c|c|c|c|c|c|}
\hline \multicolumn{6}{|c|}{ Dióxido de azufre $\left(\mathrm{SO}_{2}\right)$} \\
\hline a:motores & 182801 & 15 & 12186,7 & 0,97 & 0,5093 \\
\hline b:meses & 50020,8 & 2 & 25010,4 & 1,99 & 0,1554 \\
\hline Residuos & 365089 & 29 & 12589,3 & & \\
\hline Total (corregido) & 592762 & 46 & & & \\
\hline \multicolumn{6}{|c|}{ Monóxido de nitrógeno (no) } \\
\hline a:motores & $6,17 \cdot 10^{6}$ & 9 & 685839,0 & 12,26 & $0,0316^{\mathrm{e}}$ \\
\hline b:meses & 153278,0 & 2 & 76638,8 & 1,37 & 0,3779 \\
\hline Residuos & 167880,0 & 3 & 55960,1 & & \\
\hline Total (corregido) & $6,58 \cdot 10^{6}$ & 14 & & & \\
\hline \multicolumn{6}{|c|}{ Temperatura de los gases $\left({ }^{\circ} \mathrm{c}\right)$} \\
\hline a:motores & 6181,4 & 15 & 412,09 & 2,77 & $0,0090^{\mathrm{e}}$ \\
\hline b:meses & 384,6 & 2 & 192,3 & 1,29 & 0,2896 \\
\hline Residuos & 4309,9 & 29 & 148,62 & & \\
\hline Total (corregido) & 10958,5 & 46 & & & \\
\hline \multicolumn{6}{|c|}{ Dióxido de carbono $\left(\mathrm{co}_{2}\right)$} \\
\hline a:motores & 8,11 & 15 & 0,540479 & 0,71 & 0,7558 \\
\hline b:meses & 3,02 & 2 & 1,51341 & 1,98 & 0,1556 \\
\hline Residuos & 22,11 & 29 & 0,762466 & & \\
\hline Total (corregido) & 33,68 & 46 & & & \\
\hline \multicolumn{6}{|c|}{ Relación co/co 2} \\
\hline a:motores & 0,114732 & 15 & 0,0076488 & 1,15 & 0,3585 \\
\hline b:meses & 0,0131885 & 2 & 0,00659427 & 0,99 & 0,3824 \\
\hline Residuos & 0,192427 & 29 & 0,00663542 & & \\
\hline Total (corregido) & 0,319904 & 46 & & & \\
\hline \multicolumn{6}{|c|}{ Eficiencia de la combustión (\%) } \\
\hline a:motores & 44,0888 & 15 & 2,93925 & 0,62 & 0,8307 \\
\hline b:meses & 0,782778 & 2 & 0,391389 & 0,08 & 0,9204 \\
\hline Residuos & 136,457 & 29 & 4,70542 & & \\
\hline Total (corregido) & 181,258 & 46 & & & \\
\hline
\end{tabular}

a) Sc. Suma de cuadrados

b) Gl. Grados de libertad

c) Cm. Cuadrados medios

d) F-ratio:

e) F-valor para un $95 \%$ de probabilidad

f) Significativo para un $95 \%$ de probabilidad

Una mejor descripción del procedimiento del análisis de varianza se muestra en la figura 1. La concentración de monóxido de carbono emitida en los 3 meses evaluados indica que la combustión no se realizó de la misma forma. En el mes de mayo, las concentraciones estuvieron muy por encima comparadas con los otros 2 meses evaluados, aunque también se observaron diferencias significativas entre los meses de abril y junio. Altas concentraciones de monóxido de carbono en los gases residuales indica combustión incompleta o poscombustión, provocando la reducción de la eficiencia del motor e incremento del consumo de combustible. Aunque no fue significativa, las emisiones de dióxido de azufre, también se deben de tomar en cuenta. El valor de probabilidad de 0,1554 indica que, aunque no fue significativa la diferencia entre los meses, si 
llama la atención la variación detectada en el mes de mayo. Estas fluctuaciones en los niveles de dióxido de azufre están directamente influenciadas por las concentraciones de azufre en el combustible.
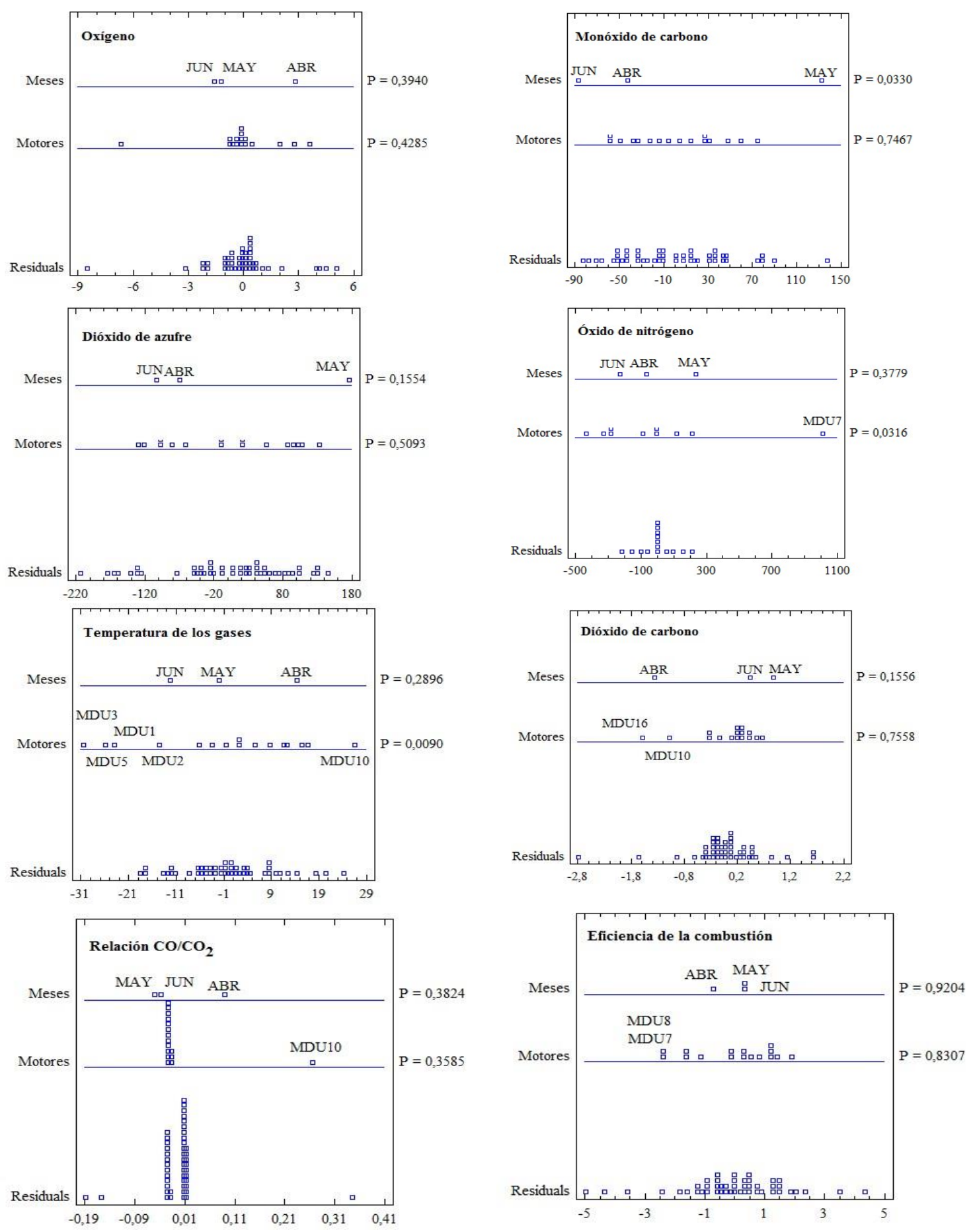

Figura 1. Anova, diagrama de los gases para las respuestas observadas. 
El combustible utilizado en los ge es de procedencia cubana, el mismo presenta altas concentraciones de azufre en su composición química. El dióxido de azufre es un gas indeseable en los motores, pues en el motor al ocurrir el proceso de combustión se incrementa la temperatura, se produce la formación de vapor de agua, y se genera el anhídrido sulfuroso (dióxido de azufre), oxidando los componentes de la cámara de combustión y conductos de escape. Otro gas que también influye en el proceso es el dióxido de carbono ( $\mathrm{p}$ valor $=0,1556$ ). Este gas, al igual que el dióxido de azufre, no fue significativo para el $95 \%$ de probabilidad, pero sus niveles si se deben de tomar en cuenta. Las concentraciones de dióxido de carbono del mes de mayo fueron superiores a los otros meses.

Utilizando el diseño experimental multifactorial, también se realizó una prueba de comparación múltiple con el objetivo de definir los motores que son diferentes en la emisión de los gases residuales estudiados (ver tabla 2).

Tabla 2

Prueba de rango múltiple y grupos homogéneos en los motores Hyundai

\begin{tabular}{|c|c|c|c|c|c|c|c|c|}
\hline \multirow[t]{2}{*}{ Motores } & \multicolumn{7}{|c|}{$\begin{array}{l}\text { Grupos } \\
\text { homogéneos }\end{array}$} & \multirow[b]{2}{*}{ Ec } \\
\hline & $\mathbf{O}_{2}$ & Co & $\mathrm{So}_{2}$ & No & $\mathrm{Tg}$ & $\mathrm{Co}_{2}$ & Co/co2 & \\
\hline Mdu1 & $x$ & $x$ & $x$ & $X x x$ & $X x$ & $X x$ & $x$ & $X$ \\
\hline Mdu2 & $x$ & $x$ & $x$ & $x x$ & $X x x$ & $X x$ & $x$ & $X$ \\
\hline Mdu3 & $x$ & $x$ & $x$ & $x$ & $X$ & $X x$ & $x$ & $X$ \\
\hline Mdu4 & $X x$ & $x$ & $x$ & $X x x$ & $x x x x$ & $X$ & $x$ & $X$ \\
\hline Mdu5 & $x$ & $x$ & $x$ & - & $X x$ & $X x$ & $x$ & $X$ \\
\hline Mdu6 & $x$ & $x$ & $x$ & - & $X x x x$ & $X$ & $x$ & $X$ \\
\hline Mdu7 & $x$ & $x$ & $x$ & $x$ & $x x x$ & $X$ & $x$ & $X$ \\
\hline Mdu8 & $x$ & $x$ & $x$ & - & $x x x x$ & $X x$ & $x$ & $X$ \\
\hline Mdu9 & $x$ & $x$ & $x$ & $X x x$ & $x x x$ & $X x$ & $x$ & $X$ \\
\hline Mdu10 & $X$ & $x$ & $x$ & $X$ & $x$ & $X x$ & $x$ & $X$ \\
\hline Mdu11 & $x$ & $x$ & $x$ & - & $x x x$ & $X x$ & $x$ & $X$ \\
\hline Mdu12 & $x$ & $x$ & $x$ & - & $x x$ & $X x$ & $x$ & $X$ \\
\hline Mdu13 & $x$ & $x$ & $x$ & - & $x x$ & $X x$ & $x$ & $X$ \\
\hline Mdu14 & $X x$ & $x$ & $x$ & $X x x$ & $x x x x$ & $X$ & $x$ & $X$ \\
\hline Mdu15 & $x$ & $x$ & $x$ & $X x x$ & $X x x x$ & $X x$ & $x$ & $X$ \\
\hline Mdu16 & $X$ & $x$ & $x$ & $X x$ & $x x x$ & $X$ & $x$ & $X$ \\
\hline
\end{tabular}

No se observaron diferencias entre los motores analizando en relación a las composiciones de dióxido de carbono, monóxido de carbono, dióxido de azufre, relación co/co2 y eficiencia de la combustión; sin embargo, sí se observaron diferencias significativas en cuanto a los gases óxido de nitrógeno, dióxido de carbono, oxígeno, y la temperatura de los gases. Una mejor representación se observa en la figura 2.

Esta variación entre los valores está en función del exceso de aire en la cámara de combustión. El exceso de aire es un factor beneficioso para la adecuada combustión del combustible, sin embargo, cuando sus niveles sobrepasan los niveles máximos, se incrementa la humedad en el sistema, se reduce la combustión y se provoca una reducción en la eficiencia, y 
por tanto se reduce la temperatura de los gases. Por otro lado, la variación en los niveles de óxidos de nitrógeno indica que los productos formados en la cámara de combustión de los motores no son similares, el cual está relacionado con el exceso de aire en la cámara, el avance al encendido, parámetros de inyección, vacío en el múltiple de admisión, temperatura en el sistema de refrigeración, entre otros, corroborando los contenidos abordados por desantes (2011).
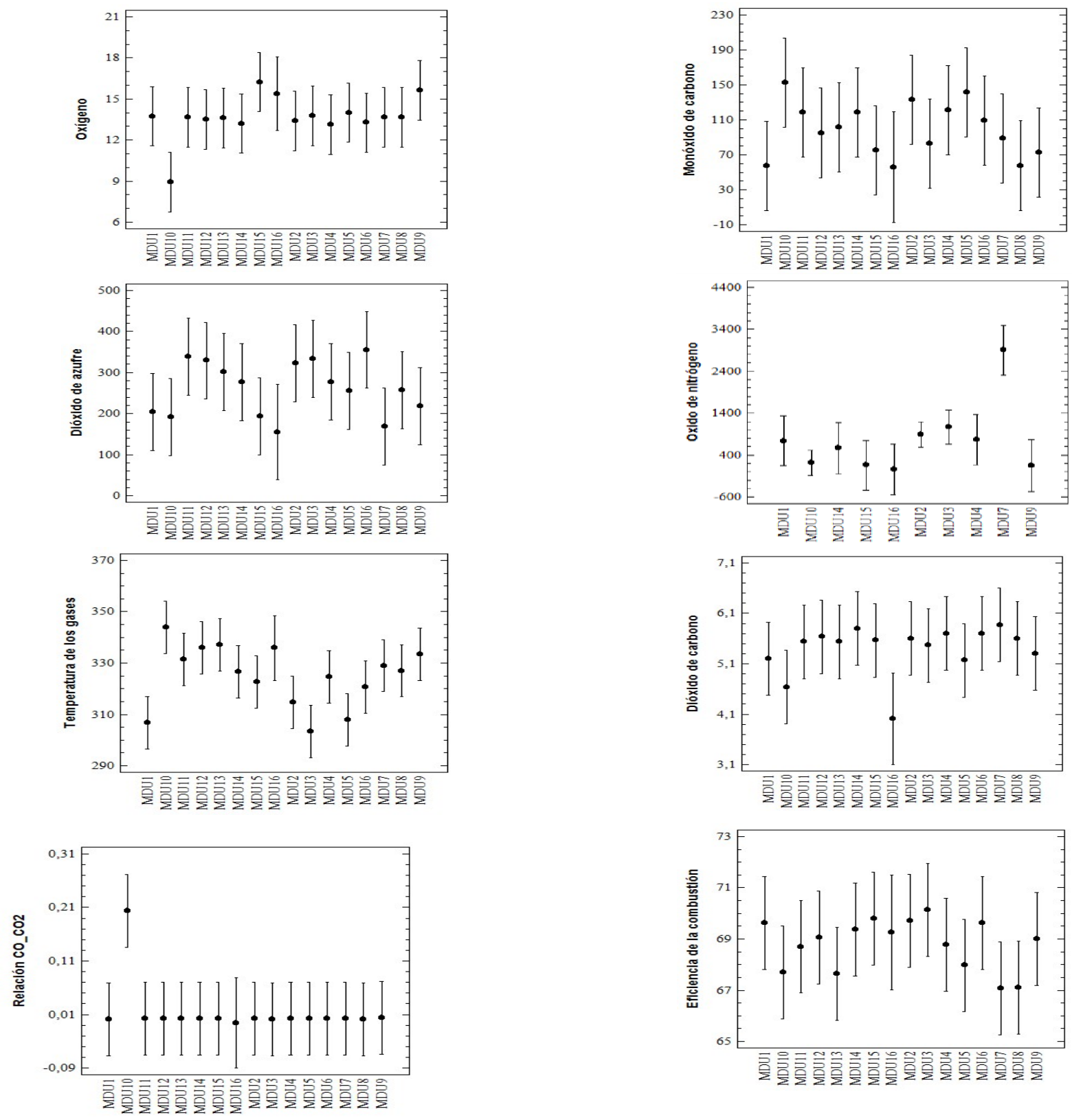

Figura 2. Rangos de medias de las respuestas observadas por motores.

En la tabla 3, se observa que, entre los 3 meses evaluados, no se observaron diferencias en los promedios de las variables medidas, excepto para el monóxido de carbono, coincidiendo con lo re-portado por la tabla 1. La variación en los niveles de monóxido de carbono reafirma que la combustión no se realiza de forma completa, influyendo en la eficiencia de operación del emplazamiento. 
Tabla 3

Prueba de rango múltiple y grupos homogéneos por meses

\begin{tabular}{llllllllll}
\hline \multirow{2}{*}{ meses } & \multicolumn{7}{c}{ Grupos homogéneos } \\
\cline { 2 - 9 } & $\mathrm{O}_{2}$ & Co & So2 & No & tg & Co2 & Co/co2 & Ec \\
\hline Abril & $\mathrm{X}$ & $\mathrm{X}$ & $\mathrm{X}$ & & $\mathrm{x}$ & $\mathrm{X}$ & $\mathrm{X}$ & $\mathrm{X}$ \\
& & & & $\mathrm{X}$ & & & & \\
Mayo & $\mathrm{X}$ & $\mathrm{X}$ & $\mathrm{X}$ & $\mathrm{X}$ & $\mathrm{x}$ & $\mathrm{X}$ & $\mathrm{X}$ & $\mathrm{X}$ \\
Junio & $\mathrm{X}$ & $\mathrm{X}$ & $\mathrm{X}$ & $\mathrm{X}$ & $\mathrm{x}$ & $\mathrm{X}$ & $\mathrm{X}$ & $\mathrm{X}$ \\
\hline
\end{tabular}

Para una mejor compresión de la variación de los niveles de las variables antes mencionadas se muestran en las figuras 3 y 4, la cual es un resumen de los gráficos de dispersión de los factores evaluados. 

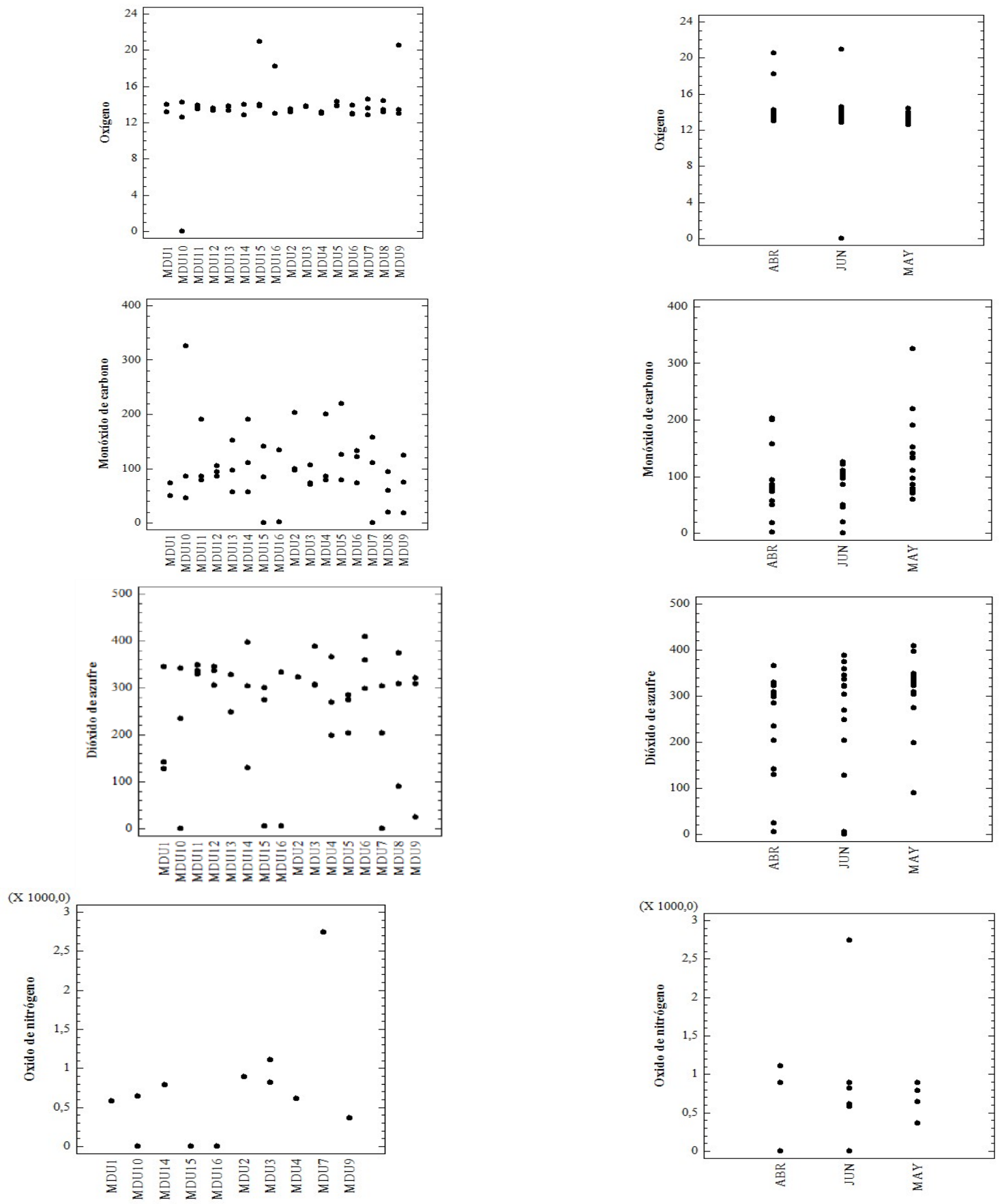

Figura 3. Gráficos de dispersión por motor y meses de evaluación 

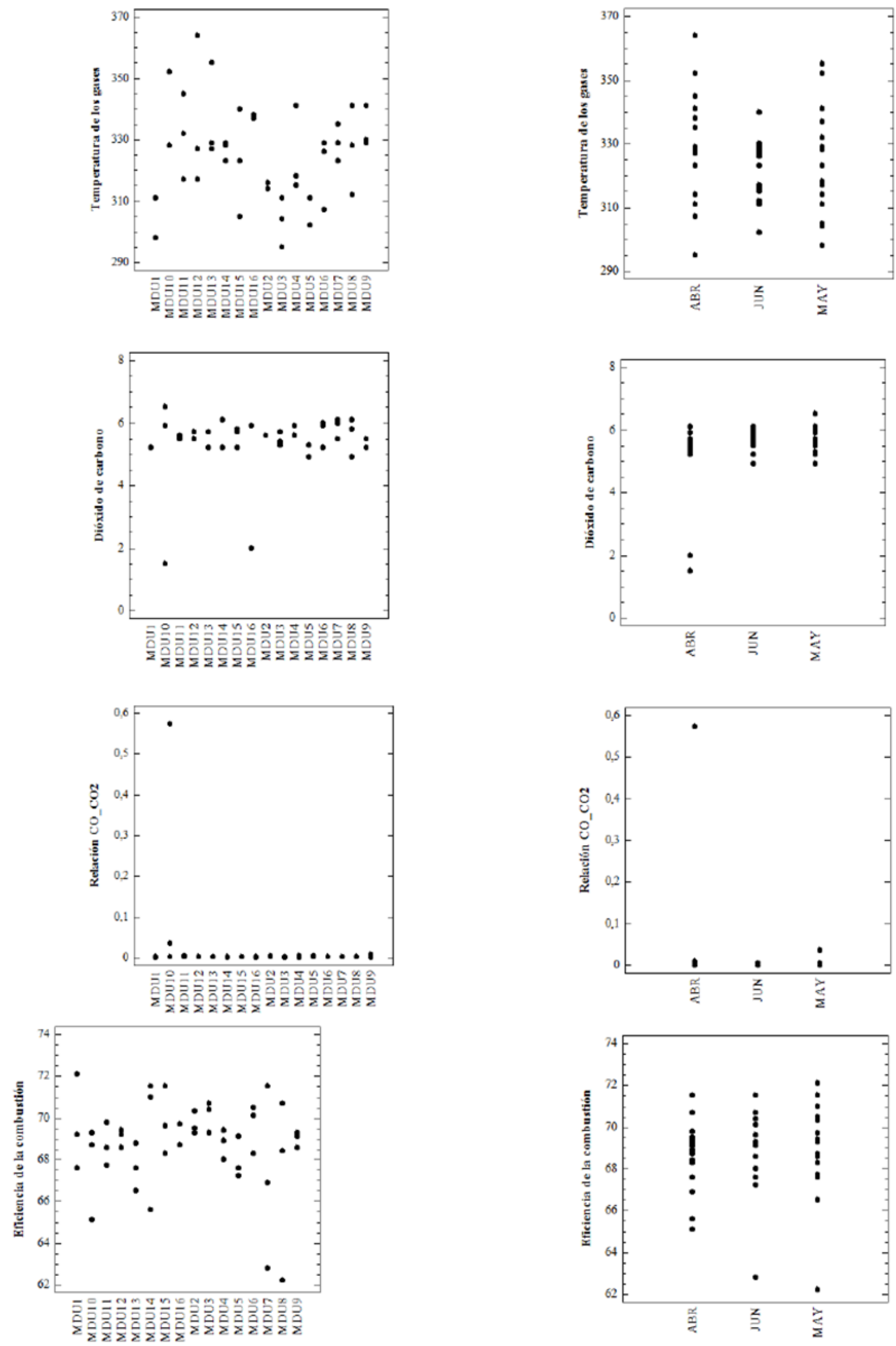

Figura 4. Gráficos de dispersión por motor y meses de evaluación (continuación). 
Analizando las emisiones de oxígeno, monóxido de carbono, óxido de nitrógeno y dióxido de carbono indican que el complejo de motores trabaja a niveles similares, excepto en los motores mdu-7, mdu-9, mdu-10, mdu-15 y mdu-16. No obstante, analizando las emisiones de dióxido de azufre y temperatura de los gases, indican una alta dispersión en los valores, evidencia de que lo motores no trabajan de forma constante. Al no ser constante la temperatura de los gases, existen variaciones en los niveles de humedad de la cámara de combustión y de la formación de compuestos químicos de fuentes de nitrógeno y azufre, y con el uso de combustibles cubanos con alto contenido de azufre, se formarán compuestos, que a la larga, provocarán la corrosión de los componentes de los motores, y por tanto un aumento en las fallas de los motores.

\section{Relación entre los gases de la combustión y las fallas de los motores}

En la figura 5 se muestran las fallas totales relacionadas con los gases de la combustión en el período evaluado por cada motor. Las fallas producidas en los 3 meses evaluados influyen significativamente en las averías producidas. Los motores que más incidencia de fallas o averías han tenido es el mdu-5, mdu-7 y mdu-15 con (3 fallas respectivamente), y seguido por el motor mdu-11 (2 fallas). Estos resultados indican que las fallas relacionadas con los gases de escape afectan en gran medida al emplazamiento de motores, pues aportan un elevado porcentaje de incidencia.

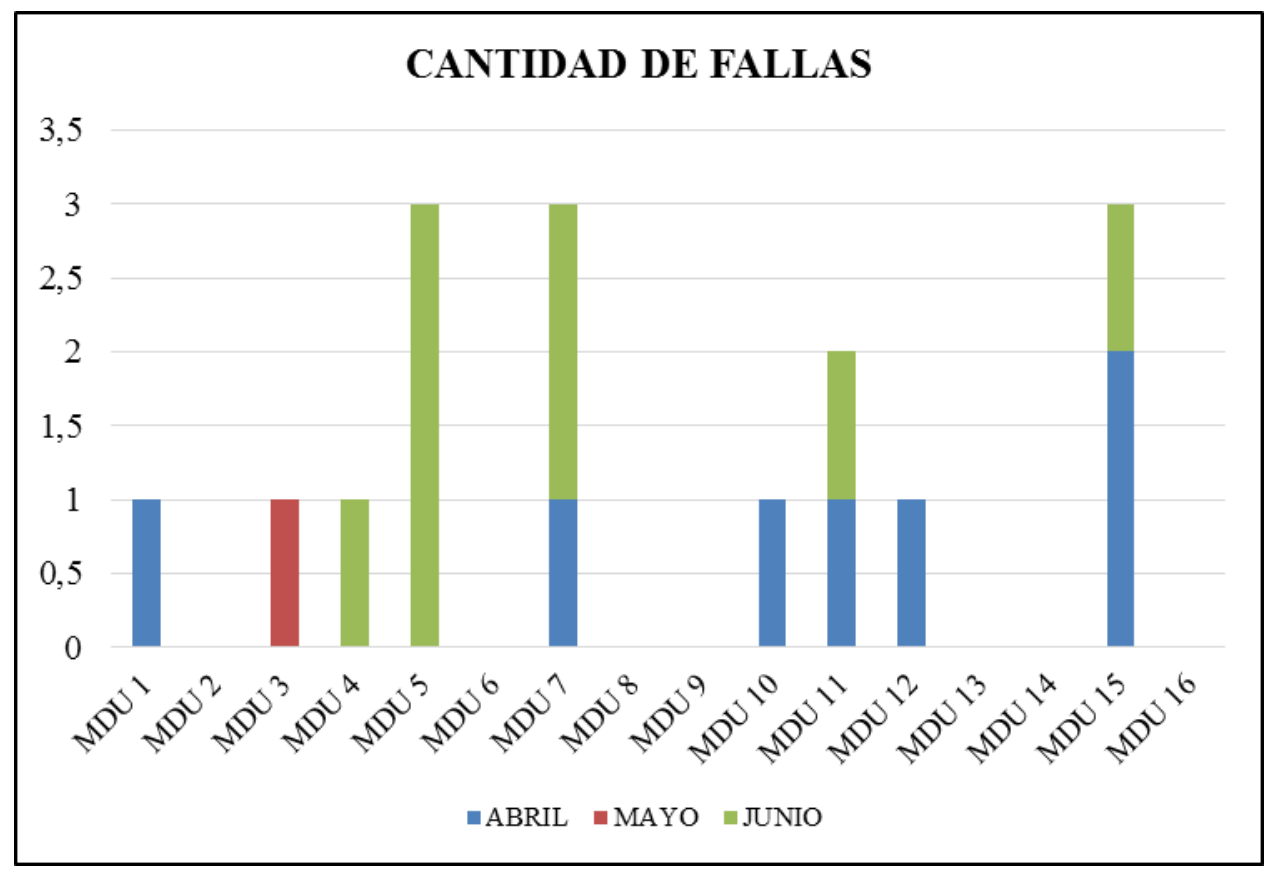

Figura 5. Fallas relacionadas con los gases de la combustión en el período evaluado en función de los motores.

Como se ha expuesto anteriormente, la temperatura de los gases influye de forma significativa en el funcionamiento de los motores; y se ha comprobado, por el análisis de la base de datos de las mediciones realizadas por la empresa, que la temperatura de combustión no es constante entre los 16 motores. De hecho, el incremento de exceso de aire por encima de lo 
permisible aumenta los niveles de nox, so2, co2, en los productos de la combustión. Todo esto contribuye a los daños de la capa de ozono por la emisión de gases de efectos invernadero. Por otro lado, el incremento de los niveles de exceso de aire induce la acumulación de los vapores de agua en la salida de los gases. La mezcla al reaccionar con el vapor de agua, obtiene iones $\mathrm{h}+$, formando compuestos sox, generando un efecto de corrosión en los equipos. Todo esto en su conjunto ayuda a la generación de fallas en el sistema.

En la figura 6 se muestra un resumen de las fallas relacionadas con los gases residuales producidos por la combustión.

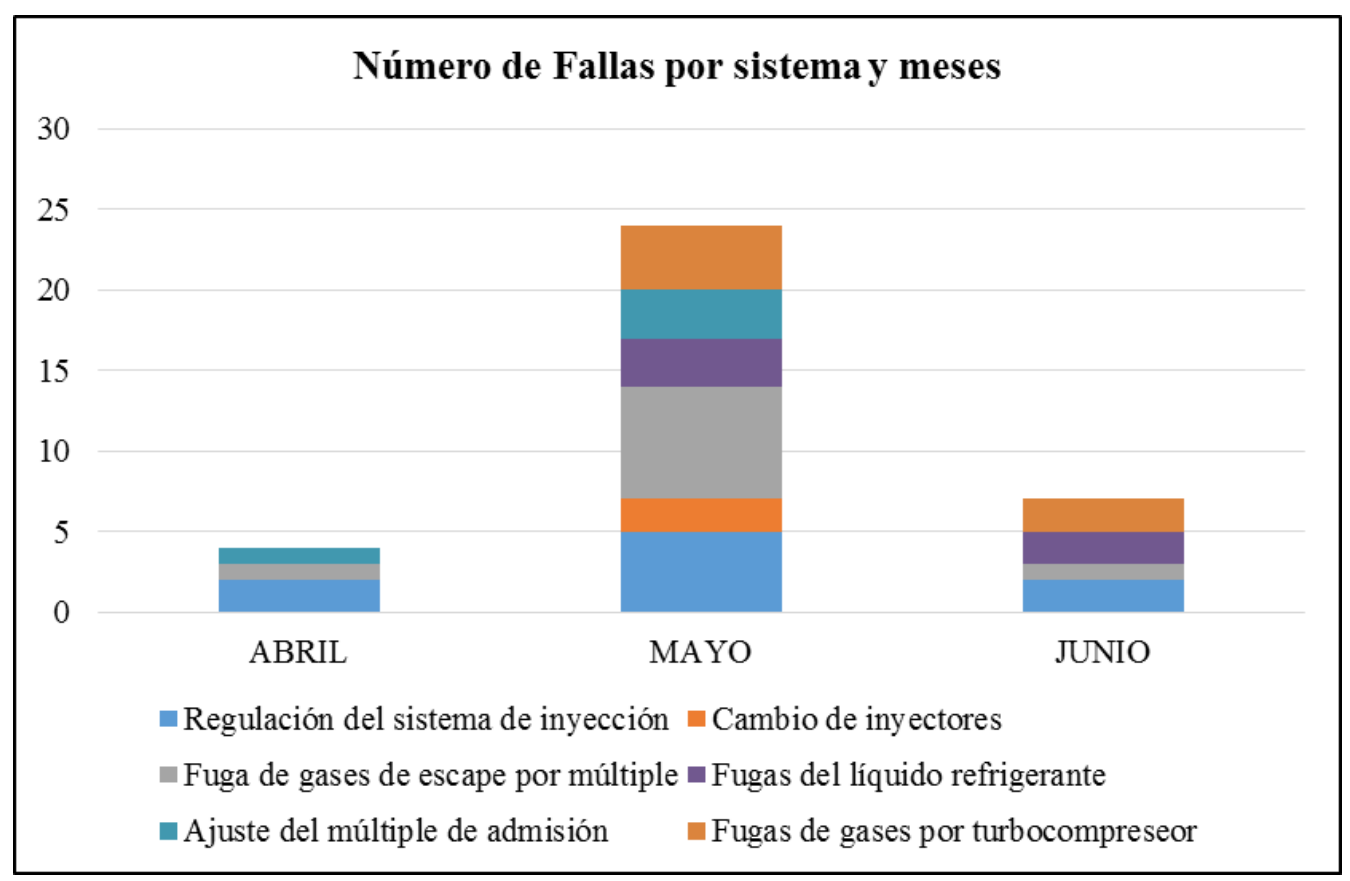

Figura 6. Fallas relacionadas con los gases residuales producidos por la combustión.

Las principales fallas se relacionan con: fugas de gases de escape por múltiple, regulación del sistema de inyección y fugas de gases por el turbocompresor. Existe una estrecha relación entre la temperatura en la cámara de combustión, la presión de los cilindros y el exceso de aire. En el proceso de escape no se logra desalojar por completo del cilindro los productos de la combustión, ocupando éstos cierto volumen a una presión y temperatura determinada. La cantidad de gases residuales depende del procedimiento empleado para limpiar el cilindro, así como la posibilidad de barrido del cilindro por la carga fresca. La temperatura depende de la composición de la mezcla, el grado de expansión y del intercambio de calor en la expansión y en el escape. En los motores diésel, donde la regulación de la carga se efectúa variando la composición de la mezcla, la temperatura decrece al disminuir la carga, provocando un aumento en la cantidad de gases residuales.

Otros factores importantes que inciden en la calidad de los gases residuales están relacionados con la calidad del combustible, tales como: la densidad específica y el peso específico, la viscosidad, y el porcentaje de impurezas del combustible. Además, inciden el 
estado de la carga, hermeticidad de la cámara de combustión, carbonización de la cámara, el aceite, entre otros.

\section{Conclusiones}

El cálculo y análisis periódico de los gases residuales de la combustión de los motores Hyundai 1,7 mw, mostró ser una herramienta efectiva que permite obtener una valoración de la calidad en la operación de los grupos electrógenos.

No se observaron diferencias entre los motores analizando en: las composiciones de dióxido de carbono, monóxido de carbono, dióxido de azufre, relación co/co2 y eficiencia de la combustión; sin embargo, sí se observaron diferencias significativas en cuanto a los gases óxido de nitrógeno, dióxido de carbono (entre los meses), y la temperatura de los gases.

El incremento de los niveles de exceso de aire induce la acumulación de los vapores de agua en la salida de los gases. La mezcla al reaccionar con el vapor de agua, obtiene iones h+, formando compuestos sox, generando un efecto de corrosión en los motores.

Las principales fallas se relacionan con fugas de gases de escape por múltiple y de escapes por el compensador. 


\section{Bibliografía}

Barelli, 1., bidini, g., buratti, c., \& mariani, r. (2009). Diagnosis of internal combustion engine through vibration and acoustic pressure non-intrusive measurements. Applied thermal engineering, 29(8), 1707-1713.

Daquinta, g. (2008). Mantenimiento y reparación de la maquinaria agrícola. Editorial félix varela, la habana, cuba.

Fernández, m. C., fernández, m. F., fuentes, r. D., \& montiel, á. C. (2011). Calidad de la energía y generación distribuida en cuba. Revista cubana de ingeniería, 1(3), 41-50.

Gardiner, d. P., allan, w. D., laviolette, m., \& bardon, m. F. (2007, january). Cycle-by-cycle exhaust temperature monitoring for detection of misfiring and combustion instability in reciprocating engines. In asme 2007 internal combustion engine division fall technical conference (pp. 573-578). American society of mechanical engineers.

Gerler, j., costin, m., fang, x., kowalczuk, z., kunwer, m., \& monajemy, r. (1995). Model based diagnosis for automotive engines-algorithm development and testing on a production vehicle. Ieee transactions on control systems technology, 3(1), 61-69.

González, f. P., \& fernández, j. M. D. (2011). Motores de combustión interna alternativos. Editorial universitat politècnica de valència.

Labis, p. E., visande, r. G., pallugna, r. C., \& caliao, n. D. (2011). The contribution of renewable distributed generation in mitigating carbon dioxide emissions. Renewable and sustainable energy reviews, 15(9), 4891-4896.

Li, w., liu, z., wang, z., xu, y., \& wang, j. (2015). Experimental and theoretical analysis of effects of $\mathrm{n} 2$, o 2 and ar in excess air on combustion and nox emissions of a turbocharged ng engine. Energy conversion and management, 97, 253-264.

San miguel, p. A. (2014). Electrotecnia. Ediciones paraninfo, sa.

Sen, cuba. (2006). Generación distribuida, una alternativa energética y económica. La habana. Cuba.

Tamura, m., saito, h., murata, y., kokubu, k., \& morimoto, s. (2011). Misfire detection on internal combustion engines using exhaust gas temperature with low sampling rate. Applied thermal engineering, 31(17), 4125-4131.

Yang, j., pu, 1., wang, z., zhou, y., \& yan, x. (2001). Fault detection in a diesel engine by analysing the instantaneous angular speed. Mechanical systems and signal processing, 15(3), 549564. 